Artículo original

\title{
Empleo del balón de contrapulsación intraaórtico como puente al trasplante cardiaco en España: resultados del estudio ASIS-TC
}

\author{
Eduardo Barge-Caballero ${ }^{\mathrm{a}, \mathrm{b}, *}$, Francisco González-Vílchez ${ }^{\mathrm{c}}$, Juan F. Delgado ${ }^{\mathrm{b}, \mathrm{d}}$, Sonia Mirabet-Pérez ${ }^{\mathrm{e}}$, \\ José González-Costello ${ }^{\mathrm{f}}$, Félix Pérez-Villa ${ }^{\mathrm{g}}$, Manuel Martínez-Sellés ${ }^{\mathrm{b}, \mathrm{h}}$, Javier Segovia-Cubero ${ }^{\mathrm{b}, \mathrm{i}}$, \\ Daniela Hervás-Sotomayor ${ }^{\mathrm{j}}$, Teresa Blasco-Peirók ${ }^{\mathrm{k}}$, Luis De la Fuente-Galán',
} José Luis Lambert-Rodríguez ${ }^{\mathrm{m}, \mathrm{n}}$, Diego Rangel-Sousa ${ }^{\mathrm{D}}$, Luis Almenar-Bonet ${ }^{\mathrm{p}}$, Iris P. Garrido-Bravo ${ }^{\mathrm{q}}$, Gregorio Rábago-Juan-Aracil ${ }^{\mathrm{r}}$, Javier Muñiz ${ }^{\mathrm{b}, \mathrm{s}}$ y María G. Crespo-Leiro ${ }^{\mathrm{a}, \mathrm{b}}$

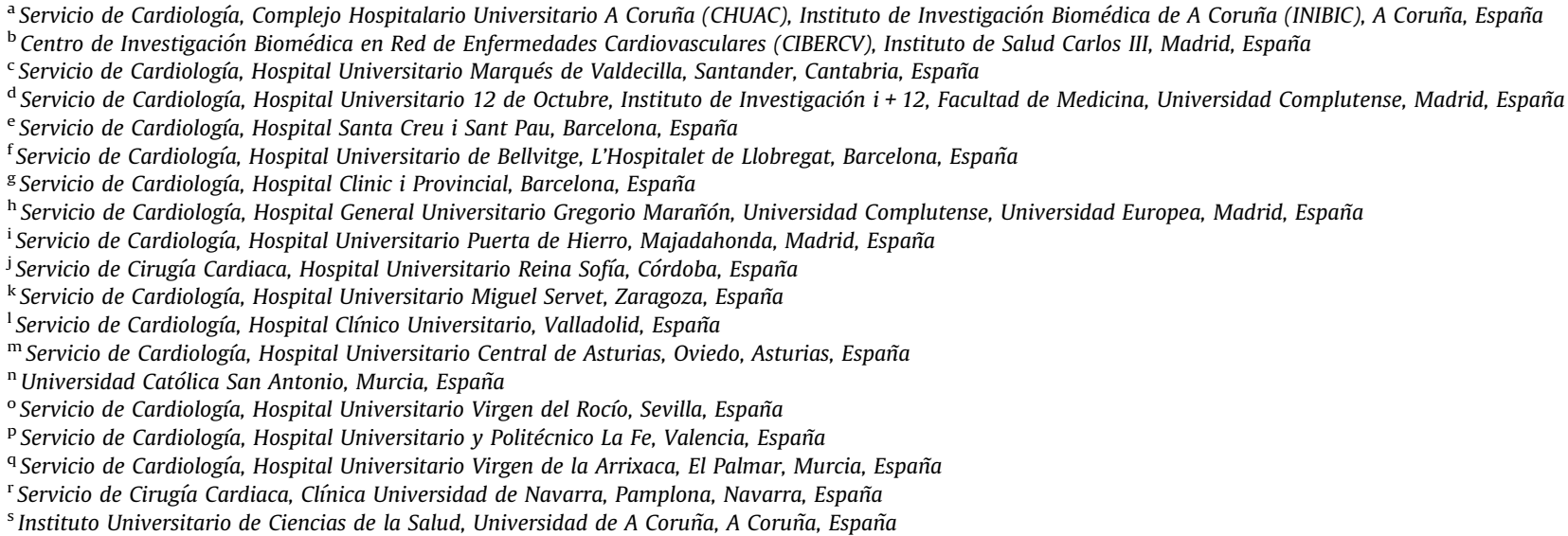

Historia del artículo:

Recibido el 9 de abril de 2018

Aceptado el 5 de julio de 2018

\section{Palabras clave:}

Balón de contrapulsación intraaórtico Asistencia circulatoria mecánica Trasplante cardiaco

\begin{abstract}
RESU M EN
Introducción y objetivos: En España, el balón de contrapulsación intraaórtico (BCIA) se ha usado frecuentemente como puente al trasplante cardiaco (TxC) urgente. El propósito es analizar los resultados de esta estrategia.

Métodos: Se realizó una revisión retrospectiva caso por caso de los registros clínicos de 281 pacientes adultos listados para TxC urgente asistidos con BCIA en 16 hospitales españoles entre 2010 y 2015. Se analizaron la supervivencia antes y después del trasplante y la incidencia de eventos adversos.

Resultados: Se trasplantó a 194 pacientes (69\%; IC95\%, 63,3-74,4) y 20 (7,1\%; IC95\%, 4,4-10,8) fallecieron durante la asistencia, cuya duración media fue de 10,9 $\pm 9,7$ días. El BCIA se explantó antes de obtener un órgano a 32 pacientes (11,4\%). En 35 pacientes (12,5\%; IC95\%, 8,8-16,9) se implantó un dispositivo de asistencia circulatoria mecánica completa. El tiempo en la lista de espera urgente se incrementó desde $5,9 \pm 6,3$ días en 2010 hasta $15 \pm 11,7$ días en $2015(\mathrm{p}=0,001)$. La supervivencia a 30 días y a 1 y 5 años tras el TxC fue del 88,1\% (IC95\%, 85,7-90,5), 76\% (IC95\%, 72,9-79,1) y 67,8\% (IC95\%, 63,7-71,9) respectivamente. La tasa de incidencia de eventos adversos mayores -disfunción del BCIA, ictus, hemorragia o infeccióndurante la asistencia fue de 26 (IC95\%, 20,6-32,4) eventos/1.000 pacientes-día. La tasa de incidencia de explante del BCIA por complicaciones fue de 7,2 (IC95\%, 4,5-10,8) casos/1.000 pacientes-día.

Conclusiones: En el contexto de listas de espera cortas, el BCIA puede utilizarse como puente al TxC urgente con resultados aceptables. Esta estrategia conlleva una incidencia significativa de eventos adversos.
\end{abstract}

(c) 2018 Sociedad Española de Cardiología. Publicado por Elsevier España, S.L.U. Todos los derechos reservados.

* Autor para correspondencia: Unidad de Insuficiencia Cardiaca Avanzada y Trasplante, Servicio de Cardiología, Complejo Hospitalario Universitario de A Coruña, As Xubias 84, 15006 A Coruña, España.

Correo electrónico: Eduardo.barge.caballero@sergas.es (E. Barge-Caballero). 
Keywords:

Intra-aortic balloon pump

Mechanical circulatory support

Heart transplant

\section{Use of Intra-aortic Balloon Pump as a Bridge to Heart Transplant in Spain: Results From the ASIS-TC Study}

A B S T R A C T

Introduction and objectives: In Spain, intra-aortic balloon pump (IABP) has been used frequently as a bridge to urgent heart transplant (HT). We sought to analyze the clinical outcomes of this strategy.

Methods: We conducted a case-by-case, retrospective review of clinical records of 281 adult patients listed for urgent HT under IABP support in 16 Spanish institutions from 2010 to 2015. Pre- and posttransplant survival and adverse clinical events were analyzed.

Results: A total of 194 (69\%, 95\%CI, 63.3-74.4) patients were transplanted and 20 (7.1\%, 95\%CI, 4.4-10.8) died during a mean period of IABP support of $10.9 \pm 9.7$ days. IABP support was withdrawn before an organ became available in $32(11.4 \%)$ patients. Thirty-five $(12.5 \%, 95 \% \mathrm{CI}, 8.8-16.9)$ patients transitioned from IABP to full-support mechanical devices. Mean urgent waiting list time increased from $5.9 \pm 6.3$ days in 2010 to $15 \pm 11.7$ days in $2015(P=.001)$. Post-transplant survival rates at 30 -days, 1 -year, and 5 -years were $88.1 \%$ (95\%CI, 85.7-90.5), 76\% (95\%CI, 72.9-79.1), and 67.8\% (95\%CI, 63.7-71.9), respectively. The incidence rate of major adverse clinical outcomes-device dysfunction, stroke, bleeding or infection-during IABP support was 26 (95\%Cl, 20.6-32.4) episodes per 1000 patient-days. The incidence rate of IABP explantation due to complications was $7.2(95 \% \mathrm{CI}, 4.5-10.8)$ cases per 1000 patient-days.

Conclusions: In a setting of short waiting list times, IABP can be used to bridge candidates to urgent HT with acceptable postoperative results, but there were significant rates of adverse clinical events during support.

(c) 2018 Sociedad Española de Cardiología. Published by Elsevier España, S.L.U. All rights reserved.

\author{
Abreviaturas \\ ACM: asistencia circulatoria mecánica \\ BCIA: balón de contrapulsación intraaórtico \\ DAV: dispositivo de asistencia ventricular \\ INTERMACS: Interagency Registry for Mechanically Assisted \\ Circulatory Support \\ TxC: trasplante cardiaco
}

\section{INTRODUCCIÓN}

El balón de contrapulsación intraaórtico (BCIA) continúa siendo en todo el mundo el dispositivo más comúnmente utilizado para proporcionar asistencia circulatoria mecánica (ACM) a los pacientes en shock cardiogénico ${ }^{1}$. Dado que la finalidad del BCIA es prestar una asistencia parcial y transitoria, es infrecuente su uso como puente directo al trasplante cardiaco (TxC). Sin embargo, en el contexto del bien organizado sistema de asignación de donantes de órganos en España $^{2}$, que garantiza un tiempo de espera corto para los candidatos a los que se asigna una prioridad de urgencia en la lista de espera, el BCIA se ha empleado con frecuencia para este fin ${ }^{3}$.

Los datos publicados hasta ahora respecto a los resultados de la asistencia con BCIA como puente directo para el TxC son escasos. El uso preoperatorio del BCIA es un factor de riesgo de muerte tras el trasplante, según la puntuación pronóstica derivada del registro de Estados Unidos ${ }^{4}$; sin embargo, en un estudio unicéntrico europeo en el que se investigó específicamente este aspecto, se observó una supervivencia tras el trasplante similar entre los candidatos a TxC que habían utilizado asistencia con BCIA como puente y los que recibieron tratamiento médico ${ }^{5}$. Hasta donde se sabe, no hay datos detallados sobre la mortalidad en lista de espera y las complicaciones clínicas asociadas con la asistencia con BCIA en esta situación de uso como puente al trasplante.

El objetivo del estudio es analizar de manera sistemática los resultados clínicos previos y posteriores al trasplante en los pacientes que recibieron asistencia mediante BCIA con la finalidad principal de servir de puente al trasplante en España y en un periodo reciente. Para ello, se analizó la información clínica incluida en un registro de ámbito nacional.

\section{MÉTODOS}

\section{Descripción del estudio}

El estudio ASIS-TC (Empleo de los dispositivos de asistencia circulatoria de corta duración como puente a trasplante cardiaco urgente en España) es un registro multicéntrico retrospectivo que incluyó a todos los pacientes de edad $\geq 18$ años incluidos entre el 1 de enero de 2010 y el 31 de diciembre de 2015 en lista de espera urgente de la Organización Nacional de Trasplantes para un primer TxC de un solo órgano estando en asistencia circulatoria de corta duración con dispositivos mecánicos (BCIA, oxigenador extracorpóreo de membrana o dispositivos de asistencia ventricular [DAV] transitoria). Participaron en el registro la totalidad de los 16 centros de $\mathrm{TxC}$ de adultos del país.

El protocolo del estudio fue aprobado por el Comité de Ética de Investigación Clínica de la Comunidad Autónoma de Galicia, y fue ratificado por los consejos de revisión internos de los hospitales participantes.

Durante el periodo de estudio, el nivel de prioridad máxima en la lista de espera para el TxC en el sistema de distribución de donantes de órganos de España (denominado urgencia 0 ) se reservó exclusivamente para los pacientes que eran incluidos en la lista de espera mientras recibían asistencia con oxigenadores de membrana extracorpóreos o DAV transitorios, o los pacientes con DAV de uso prolongado que presentaban un mal funcionamiento. El urgencia 0 conlleva la prioridad de ámbito nacional para recibir el primer órgano de donante adecuado que esté disponible en el sistema. En un estudio reciente se han descrito los resultados clínicos de los candidatos a trasplante en urgencia $0^{6}$.

A los candidatos incluidos en lista de espera para TxC asistidos con un BCIA se los incluyó en un nivel de prioridad inferior (denominado urgencia 1), que implica una mayor prioridad que la de los candidatos en tratamiento médico, incluidos en urgencia 2 (es decir, el nivel de intervención electiva o no urgente) para la recepción de un corazón de donante adecuado, siempre que no haya ningún candidato urgencia 0 que pueda utilizarlo. Hasta junio de 2014, la prioridad de los candidatos en urgencia 1 respecto a los de urgencia 2 se aplicó a todos los órganos obtenidos en todo el país, pero a partir de esa fecha solamente se aplica a los obtenidos en el área de asistencia correspondiente al centro de trasplante donde el candidato está hospitalizado. 
En este estudio se presentan los resultados clínicos obtenidos en los pacientes incluidos en lista de espera para TxC mientras recibían asistencia circulatoria con un BCIA como único dispositivo de ACM, y por lo tanto clasificados como casos de urgencia 1. En el caso de que un paciente hubiera estado en lista de espera para TxC en urgencia 1 en más de una ocasión durante el periodo del estudio, se tuvo en cuenta solo el episodio más reciente de inclusión en la lista. Cada equipo de trasplante adoptó la decisión de implantar un BCIA y, posteriormente, incluir al paciente en la lista de espera para TxC como urgencia 1 según los protocolos y la experiencia clínica locales, pero no se basó en un protocolo preespecificado definido por el estudio.

Se analizaron las características clínicas basales, los resultados y las complicaciones de la asistencia circulatoria con BCIA, los tiempos de espera y los resultados tras el trasplante. Las definiciones específicas de los criterios de valoración del estudio se presentan en el material suplementario.

\section{Análisis estadístico}

Las variables continuas se presentan como media \pm desviación estándar y las cualitativas, como proporciones. Se utilizaron la prueba de la $t$ de Student y la prueba de la $\chi^{2}$ para las comparaciones estadísticas entre los grupos, según fuera necesario. Se utilizó el método exacto de Clopper-Pearson para calcular los intervalos de confianza del 95\% (IC95\%) de las tasas de incidencia de los criterios de valoración del estudio. Se utilizó un análisis de la varianza de una vía con contraste polinómico lineal para analizar la tendencia de los tiempos de permanencia en lista de espera para el TxC a lo largo del periodo de estudio.

Se elaboraron curvas de Kaplan-Meier de la supervivencia tras la inclusión en lista de espera y tras el trasplante, y se compararon mediante log-rank test. Se utilizó una regresión multivariable de Cox para estimar el valor de la hazard ratio de la mortalidad a 1 año tras la inclusión en lista de espera urgente de los pacientes con los perfiles 1-2 frente a los perfiles 3-4 del Interagency Registry for Mechanically Assisted Circulatory Support (INTERMACS), con un ajuste respecto a edad, sexo y etiología de la insuficiencia cardiaca (isquémica o no isquémica). La significación estadística se definió por un valor de $\mathrm{p}<0,05$ en todas las comparaciones. Los análisis estadísticos se realizaron con los programas SPSS 20 y Epidat 4.1.

\section{RESULTADOS}

\section{Pacientes}

Entre enero de 2010 y diciembre de 2015, se incluyó en la lista de espera para $\mathrm{TxC}$ a 281 pacientes con asistencia circulatoria mediante BCIA, y por lo tanto con prioridad de urgencia 1, en 16 hospitales de España. Un total de 138 (49,1\%) de estos candidatos ya estaban incluidos en la lista de espera para $\mathrm{TxC}$ antes del implante del BCIA, y se había elevado su prioridad a urgencia 1 tras la colocación de este dispositivo. Durante el periodo del estudio, 3 pacientes ingresaron en más de 1 ocasión en la lista de espera para TxC en urgencia 1 por estar en asistencia con BCIA.

El BCIA se implantó a través de la arteria femoral a todos los pacientes excepto $2(0,7 \%)$, para los que se optó por acceso humeral. El calibre del dispositivo fue $7 \mathrm{Fr}$ en 89 pacientes (32\%), 8 Fr en 159 (57\%) y 9 Fr en 23 (8\%); en 10 pacientes no se consignó este dato. La media de tiempo transcurrido entre el implante del BCIA y la inclusión en lista de espera en urgencia 1 fue de 1,7 \pm 3,6 días.

En la tabla 1 se presentan las principales características clínicas basales de los pacientes del estudio, según la evaluación realizada en el momento de la inclusión en lista de espera en urgencia 1.
Tabla 1

Características clínicas basales de los pacientes del estudio en el momento de la inclusión en urgencia 1

\begin{tabular}{|c|c|}
\hline \multicolumn{2}{|l|}{ Variables } \\
\hline \multicolumn{2}{|l|}{ Antecedentes } \\
\hline Edad (años) & $52,9 \pm 11$ \\
\hline Mujeres & $65(23,1)$ \\
\hline Índice de masa corporal & $25,8 \pm 10,3$ \\
\hline Estancia hospitalaria hasta uso de BCIA (días) & $14,9 \pm 19,9$ \\
\hline Tiempo desde implante de BCIA hasta inclusión en urgencia 1 & $1,7 \pm 3,6$ \\
\hline Pacientes en lista de espera antes del implante del BCIA & $138(49,1)$ \\
\hline \multicolumn{2}{|l|}{ Etiología de la miocardiopatía subyacente } \\
\hline Isquémica & $119(42,3)$ \\
\hline Dilatada (idiopática/familiar) & $114(40,6)$ \\
\hline Hipertrófica & $14(5,0)$ \\
\hline Valvular & $9(3,2)$ \\
\hline Miocarditis & $7(2,5)$ \\
\hline Restrictiva & $5(1,8)$ \\
\hline Tras quimioterapia & $4(1,5)$ \\
\hline Arritmogénica & $3(1,1)$ \\
\hline Congénita & $3(1,1)$ \\
\hline No compactada & $3(1,1)$ \\
\hline Shock cardiogénico relacionado con infarto agudo de miocardio & $42(14,9)$ \\
\hline Shock cardiogénico tras cirugía cardiaca & $2(0,7)$ \\
\hline Diabetes mellitus & $64(22,8)$ \\
\hline Hipertensión & $97(34,5)$ \\
\hline Hipercolesterolemia & $106(37,7)$ \\
\hline Cirugía cardiaca abierta previa & $42(14,9)$ \\
\hline Antecedentes de cáncer & $8(2,8)$ \\
\hline Enfermedad vascular periférica & $11(3,9)$ \\
\hline Enfermedad pulmonar obstructiva crónica & $39(13,9)$ \\
\hline Ictus previo & $22(7,8)$ \\
\hline Antecedentes de arritmia ventricular & $115(40,9)$ \\
\hline Antecedentes de fibrilación auricular & $118(42,0)$ \\
\hline Parada cardiaca previa & $40(14,2)$ \\
\hline Desfibrilador implantable & $158(56,2)$ \\
\hline Terapia de resincronización cardiaca & $54(19,2)$ \\
\hline Infección activa que requiere tratamiento intravenoso & $23(8,2)$ \\
\hline \multicolumn{2}{|l|}{ Estado clínico } \\
\hline \multicolumn{2}{|l|}{ Perfil de INTERMACS } \\
\hline INTERMACS 1 & $51(18,1)$ \\
\hline INTERMACS 2 & $126(44,8)$ \\
\hline INTERMACS 3 & $79(28,9)$ \\
\hline INTERMACS 4 & $19(6,8)$ \\
\hline No indicado & $6(2,1)$ \\
\hline Presión arterial sistólica $(\mathrm{mmHg})$ & $99 \pm 13$ \\
\hline Frecuencia cardiaca (lpm) & $88 \pm 19$ \\
\hline \multicolumn{2}{|l|}{ Tratamientos de apoyo } \\
\hline$B C I A$ & $281(100)$ \\
\hline Terapia de sustitución renal & $6(2,1)$ \\
\hline Ventilación mecánica & $42(14,9)$ \\
\hline Inotrópicos & $209(74,4)$ \\
\hline Vasopresores & $53(18,9)$ \\
\hline Puntuación vasoactiva-inotrópica (unidades) & $16 \pm 42$ \\
\hline \multicolumn{2}{|l|}{ Análisis de laboratorio } \\
\hline Leucocitos $\left(\times 10^{9} / l\right)$ & $9,2 \pm 4,5$ \\
\hline Plaquetas $\left(\times 10^{9} / l\right)$ & $190 \pm 100$ \\
\hline INR & $1,5 \pm 0,7$ \\
\hline Tiempo de protrombina $(\mathrm{s})$ & $21 \pm 15$ \\
\hline
\end{tabular}


Tabla 1 (Continuación)

Características clínicas basales de los pacientes del estudio en el momento de la inclusión en urgencia 1

\begin{tabular}{|c|c|}
\hline Variables & \\
\hline Creatinina $(\mathrm{mg} / \mathrm{dl})$ & $1,2 \pm 0,5$ \\
\hline Tasa de filtrado glomerular ( $\mathrm{ml} / \mathrm{min}$ ) & $78 \pm 32$ \\
\hline Hemoglobina $(\mathrm{g} / \mathrm{dl})$ & $11,6 \pm 2,1$ \\
\hline Sodio $(m E q / l)$ & $135 \pm 5$ \\
\hline Bilirrubina $(\mathrm{mg} / \mathrm{dl})$ & $1,9 \pm 2,2$ \\
\hline Aspartato aminotransferasa (UI/l) & $62 \pm 96$ \\
\hline Alanina aminotransferasa $(U I / l)$ & $79 \pm 139$ \\
\hline Albúmina $(\mathrm{g} / \mathrm{dl})$ & $3,7 \pm 0,7$ \\
\hline Presión arterial de oxígeno $(\mathrm{mmHg})$ & $103 \pm 43$ \\
\hline$p H$ & $7,44 \pm 0,1$ \\
\hline Lactato $(\mathrm{mmol} / \mathrm{l})$ & $1,5 \pm 1,2$ \\
\hline \multicolumn{2}{|l|}{ Ecocardiografía } \\
\hline Fracción de eyección del ventrículo izquierdo (\%) & $23 \pm 9$ \\
\hline Diámetro telesistólico del ventrículo izquierdo ( $\mathrm{mm}$ ) & $66 \pm 11$ \\
\hline Desplazamiento sistólico del anillo tricuspídeo ( $\mathrm{mm}$ ) & $14 \pm 4$ \\
\hline \multicolumn{2}{|l|}{ Hemodinámica } \\
\hline Índice cardiaco $\left(\mathrm{ml} / \mathrm{min} / \mathrm{m}^{2}\right)$ & $2,3 \pm 0,7$ \\
\hline Presión venosa central ( $\mathrm{mmHg})$ & $13 \pm 6$ \\
\hline Presión capilar enclavada ( $\mathrm{mmHg}$ ) & $23 \pm 8$ \\
\hline Presión pulmonar media ( $\mathrm{mmHg}$ ) & $33 \pm 11$ \\
\hline Gradiente transpulmonar ( $\mathrm{mmHg}$ ) & $10 \pm 5$ \\
\hline
\end{tabular}

BCIA: balón de contrapulsación intraaórtico; INTERMACS: Interagency Registry for Mechanically Assisted Circulatory Support.

Los valores expresan media \pm desviación estándar o n (\%).

\section{Resultados de la asistencia con balón de contrapulsación intraaórtico}

La media de duración de la asistencia con BCIA fue de 10,9 $\pm 9,7$ (intervalo, 0-58) días. Durante ese tiempo, se realizó el reemplazo programado del dispositivo (es decir, no debido a complicaciones) en 1 ocasión en 5 pacientes y en 2 ocasiones en 2 pacientes. En la figura 1 se presenta un diagrama de flujo de los pacientes del estudio y los resultados obtenidos con la asistencia circulatoria con BCIA.

En total, se trasplantó a 194 pacientes (69\%; IC95\%, 63,3-74,4) y 20 pacientes (7,1\%; IC95\%, 4,4-10,8) fallecieron durante la asistencia con BCIA. La media del tiempo de espera para el TxC fue de $9,6 \pm 10$ días, con un aumento constante a lo largo del periodo de estudio, en el que pasó de 5,9 \pm 6,3 días en 2010 a $15 \pm 11,7$ días en 2015 (figura 2; p de tendencia lineal $=0,001$ ).

En 35 pacientes (12,5\%; IC95\%, 8,8-16,9) se pasó del BCIA a una asistencia circulatoria completa con dispositivos mecánicos antes de que se dispusiera de un donante. Los dispositivos implantados fueron oxigenadores de membrana extracorpóreos venoarteriales $(n=14)$, Levitronix Centrimag $(n=13)$, Impella Recover $(n=4)$, Abiomed BVS $5000(n=3)$ y BerlinHeart Excor $(n=1)$. De estos pacientes, 11 fallecieron durante la ACM y 23 recibieron un trasplante después como candidatos en urgencia 0 ( 8 de ellos fallecieron en el posoperatorio inmediato al $\mathrm{TxC}$ ). Un paciente al que se implantó un dispositivo BerlinHeart Excor fue dado de alta del hospital con este dispositivo y 3 meses después recibió el trasplante con éxito.

La asistencia circulatoria con BCIA se retiró antes de que se dispusiera de un donante en 32 pacientes (11,4\%; IC95\%, 7,9-15,7), a los que después se dio tratamiento médico. Las razones para cesar la asistencia circulatoria con BCIA fueron complicaciones en 19 pacientes, una mejoría clínica en 10 y futilidad en 3. Durante la estancia hospitalaria tras el cese de la asistencia con BCIA, a 18 pacientes se les practicó un TxC (todos ellos sobrevivieron a la operación) y 7 fallecieron sin llegar al trasplante.

En total, se practicó un TxC durante el periodo de seguimiento en el hospital tras su inclusión en la lista de espera como urgencia 1 a 235 pacientes (83,6\%; IC95\%, 78,9-87,8); 39 pacientes $(13,9 \%$; IC95\%, 10,1-18,5) fallecieron durante el ingreso hospitalario sin recibir el trasplante.

En la figura 3 se muestra la frecuencia con que se produjeron eventos en competencia de TxC durante la asistencia con BCIA, muerte durante la asistencia con BCIA, transición a ACM completa o retirada del BCIA (y paso a tratamiento médico) en la población en estudio durante un periodo de seguimiento de 28 días tras la inclusión en la lista como urgencia 1.

\section{Eventos adversos clínicos durante la asistencia con balón de contrapulsación intraaórtico}

La tasa de incidencia de eventos adversos clínicos durante la asistencia con BCIA fue de 38,7 (IC95\%, 32,1-46,3) eventos/ 1.000 días-paciente; la tasa de incidencia de eventos adversos

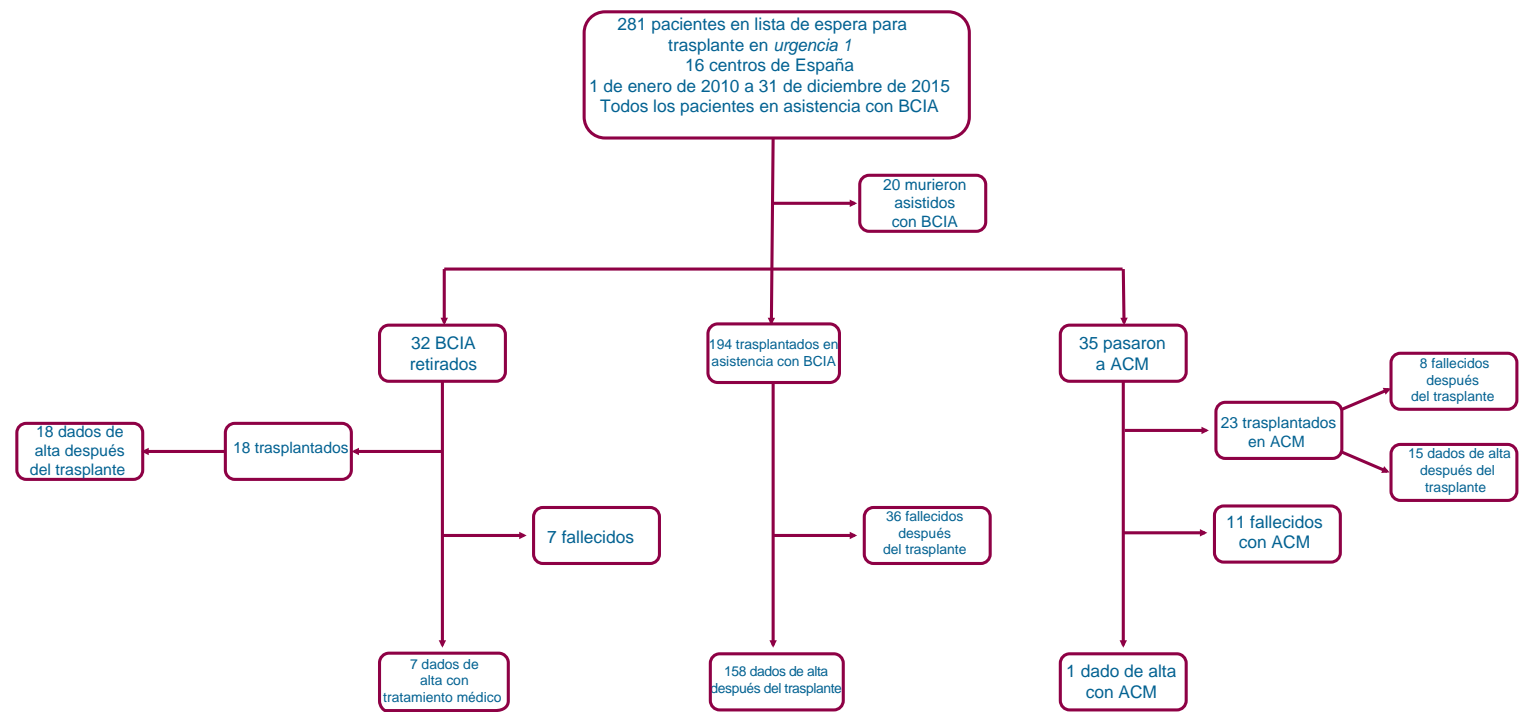

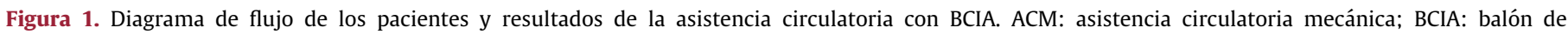
contrapulsación intraaórtico. 


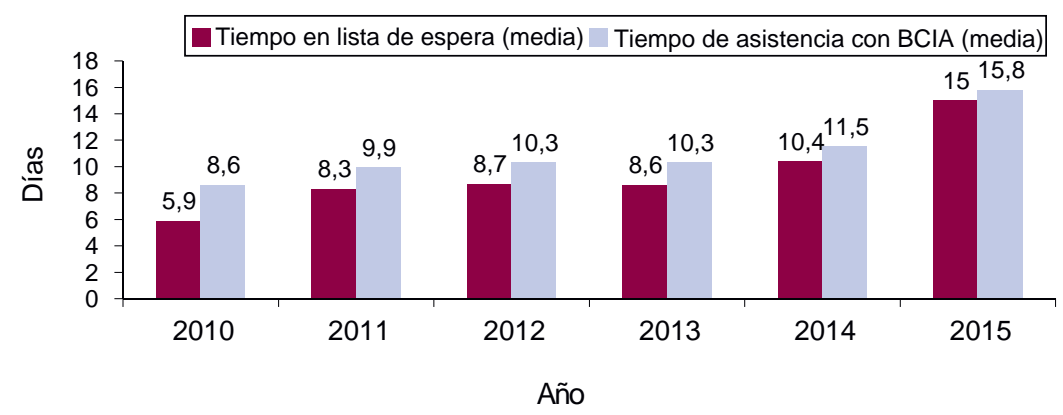

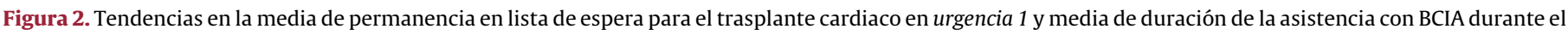
periodo de estudio. BCIA: balón de contrapulsación intraaórtico.

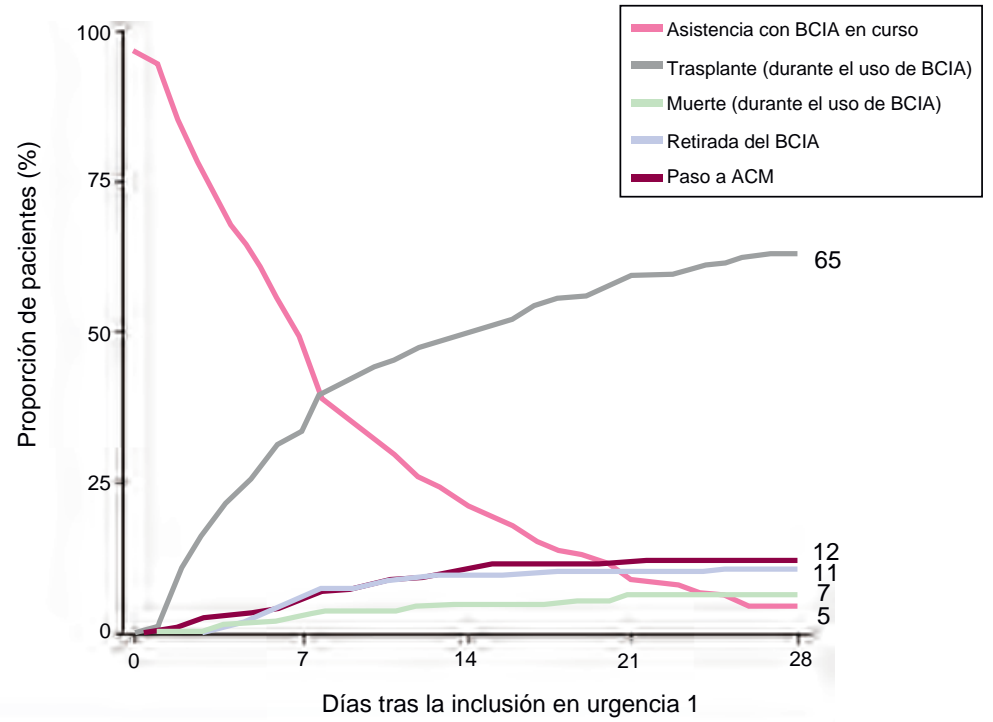

\begin{tabular}{lccccc}
\hline Asistencia con BCIA en curso & 281 & 143 & 63 & 26 & 13 \\
Trasplante durante el uso de BCIA & 0 & 97 & 145 & 173 & 183 \\
Muerte durante el uso de BCIA & 0 & 9 & 14 & 18 & 19 \\
Retirada del BCIA & 0 & 16 & 28 & 30 & 31 \\
Paso a ACM & 0 & 16 & 31 & 34 & 35 \\
\hline
\end{tabular}

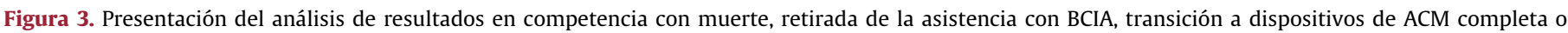

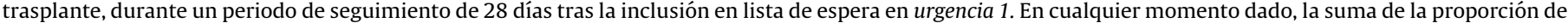
pacientes que presentan cada uno de los resultados es igual al 100\%. ACM: asistencia circulatoria mecánica; BCIA: balón de contrapulsación intraaórtico.

clínicos mayores (hemorragia mayor, ictus, infección o mal funcionamiento del BCIA) fue de 26,0 (IC95\%, 20,6-32,4) eventos/ 1.000 días-paciente. En la tabla 2 se muestra la tasa de incidencia de cada uno de los eventos adversos clínicos registrados en el estudio.

Se explantó el BCIA a causa de complicaciones relacionadas con el dispositivo en 22 pacientes (7,8\%) antes de que se dispusiera de un órgano para el trasplante; en 3 de estos pacientes se volvió a implantar un nuevo BCIA. Las razones para el explante del BCIA fueron la isquemia/tromboembolia arterial $(n=11)$, el mal funcionamiento del dispositivo $(n=5)$, una infección $(n=4)$ o un dolor resistente al tratamiento $(n=2)$. La tasa de incidencia de explantes del BCIA a causa de complicaciones asociadas con el dispositivo fue de 7,2 (IC95\%, 4,5-10,8) casos/1.000 días-paciente.

\section{Resultados tras el trasplante}

De los 194 pacientes sometidos a TxC urgente estando en asistencia con BCIA, 36 (18,6\%; IC95\%, 13,3-24,7) fallecieron en el posoperatorio en el hospital. En la tabla 3 se muestran las tasas de incidencia de otros resultados posoperatorios hospitalarios tras el
TxC. La media del tiempo de isquemia fría de estas intervenciones fue de $213 \pm 52 \mathrm{~min}$. La media de edad de los donantes fue de $42 \pm 12,6$ años. Se utilizaron donantes de edad $\geq 45$ años y donantes con un tiempo de isquemia $\geq 240 \mathrm{~min}$ en $93(47,9 \%)$ y 66 (34\%) receptores respectivamente.

Las causas de muerte posoperatoria en el hospital tras el TxC fueron fallo temprano del injerto $(n=17)$, infección $(n=7)$, hemorragia quirúrgica $(\mathrm{n}=5)$, rechazo $(\mathrm{n}=3)$, muerte súbita $(\mathrm{n}=1)$ y fallo multiorgánico no especificado $(\mathrm{n}=3)$.

Se estimaron con el método de Kaplan-Meier las tasas de supervivencia a 30 días y 1 y 5 años tras el TxC, que fueron del 88,1\% (IC95\%, 85,7-90,5), 76\% (IC95\%, 72,9-79,1) y 67,8\% (IC95\%, 63,7-71,9) respectivamente (figura 4).

\section{Supervivencia general tras la inclusión en lista de trasplante en urgencia 1}

La supervivencia general a 1 año tras la inclusión en la lista de espera en urgencia 1, teniendo en cuenta tanto el periodo de uso de asistencia como el periodo tras el trasplante (si había tenido 
Tabla 2

Eventos adversos clínicos durante la asistencia con balón de contrapulsación intraaórtico

\begin{tabular}{|c|c|c|}
\hline Evento adverso clínico & $\mathrm{n}(\%)$ & Tasa de incidencia (IC95\%) \\
\hline Infección & $71(25,3)$ & $23,1(18,0-29,1)$ \\
\hline Infección relacionada con el $\mathrm{BCIA}$ & $5(1,8)$ & $1,6(0,5-3,8)$ \\
\hline Hemorragia mayor & $10(3,6)$ & $3,3(1,6-6,0)$ \\
\hline Ictus & $2(0,7)$ & $0,6(0,1-2,3)$ \\
\hline Tromboembolia arterial fuera del SNC & $15(5,3)$ & $4,9(2,7-8,0)$ \\
\hline Tromboembolia venosa & $3(1,1)$ & $1(0,2-2,8)$ \\
\hline Insuficiencia renal que requiere terapia de sustitución renal & $17(6)$ & $5,5(3,2-8,8)$ \\
\hline Derrame pleural que requiere drenaje & $3(1,1)$ & $1(0,2-2,8)$ \\
\hline Derrame pericárdico que requiere drenaje & $3(1,1)$ & $1(0,2-2,8)$ \\
\hline Hemolisis & $4(1,4)$ & $1,3(0,4-3,3)$ \\
\hline Complicación en lugar de acceso vascular & $26(9,3)$ & $8,5(5,5-12,4)$ \\
\hline Isquemia de extremidades & $19(6,8)$ & $6,2(3,7-9,7)$ \\
\hline Hematoma & $6(2,2)$ & $1,9(0,7-4,2)$ \\
\hline Dolor resistente al tratamiento & $2(0,7)$ & $0,6(0,1-2,3)$ \\
\hline Infección & $1(0,4)$ & $0,3(0,0-0,8)$ \\
\hline Seudoaneurisma & $1(0,4)$ & $0,3(0,0-0,8)$ \\
\hline Fístula arteriovenosa & 0 & 0 \\
\hline Disfunción del BCIA & $7(2,5)$ & $2,3(0,9-4,7)$ \\
\hline Algún evento adverso mayor & $80(28,5)$ & $26(20,6-32,4)$ \\
\hline Algún evento adverso & $119(42,3)$ & $38,7(32,1-46,3)$ \\
\hline
\end{tabular}

BCIA: balón de contrapulsación intraaórtico; IC95\%: intervalo de confianza del 95\%; SNC: sistema nervioso central.

La tasa de incidencia es el número de pacientes con un evento cada 1.000 días-paciente de asistencia.

lugar) o el periodo tras la asistencia (si no se trasplantó) fue del $66,7 \%$ (IC95\%, 63,9-69,5). La hazard ratio ajustada de la mortalidad por cualquier causa a 1 año tras la inclusión en la lista de espera en urgencia 1 de los pacientes con perfiles de INTERMACS 1-2 frente a los de perfiles 3-4, ajustada por edad, sexo y etiología de la insuficiencia cardiaca, fue de 2,17 (IC95\%, 1,32-3,58; p=0,005) (figura $5 \mathrm{~A}$ ). Los candidatos con INTERMACS 1-2 presentaron unas tasas superiores de transición al uso de dispositivos de asistencia circulatoria completa (el 16,9 frente al $4,1 \% ; \mathrm{p}=0,002$ ) y unas tasas de trasplante inferiores (el 65 frente al 78,6\%; $\mathrm{p}=0,019$ ) durante la asistencia con BCIA, en comparación con los candidatos con INTERMACS 3-4.
La supervivencia a 1 año tras la inclusión en lista de espera en urgencia 1 mostró diferencias significativas ( $p=0,001$ ) según el objetivo clínico de la asistencia con BCIA (figura $5 \mathrm{~B}$ ). Los pacientes que pasaron del BCIA a un dispositivo de ACM completa fueron los que obtuvieron peor resultado, con una supervivencia estimada a 1 año tras la inclusión en lista de espera en urgencia 1 del 42,9\% (IC95\%, 34,5-51,3). La supervivencia estimada a 1 año tras la inclusión en lista de espera en urgencia 1 fue del 78,1\% (IC95\%, 70,8$85,4)$ de los pacientes que cesaron la asistencia con BCIA y después recibieron tratamiento médico; esta cifra es comparable a la supervivencia de los pacientes sometidos a trasplante directamente con el BCIA. Las curvas de supervivencia a 1 año tras el

Tabla 3

Resultados posoperatorios hospitalarios tras el trasplante cardiaco en 194 pacientes con balón de contrapulsación intraaórtico como puente

\begin{tabular}{lll}
\hline & $\mathrm{n}(\%)$ & Tasa de incidencia (IC95\%) \\
\hline Eventos adversos posoperatorios & & $10,1(7,8-13,0)$ \\
\hline Fallo primario del injerto & $62(32)$ & $6,2(4,4-8,5)$ \\
\hline$\quad$ Ventricular derecho & $38(19,6)$ & $3,9(2,5-5,8)$ \\
\hline$\quad$ Ventricular izquierdo o biventricular & $24(12,4)$ & $2,3(1,2-3,8)$ \\
\hline ACM transitoria tras el trasplante & $14(7,2)$ & $6,9(4,9-9,3)$ \\
\hline Hemorragia quirúrgica excesiva & $42(21,6)$ & $7,8(5,8-10,4)$ \\
\hline Reintervención mediante cirugía abierta & $48(24,7)$ & $13,6(10,8-16,8)$ \\
\hline Infección posoperatoria & $83(42,8)$ & $9,2(6,9-11,9)$ \\
\hline Insuficiencia renal que requiere terapia de sustitución & $56(28,9)$ & $5,9(4,1-8,1)$ \\
\hline Muerte posoperatoria durante la hospitalización & $36(18,6)$ & \\
\hline Otros resultados posoperatorios & & \\
\hline Respiración asistida después del trasplante (días) & $6,1 \pm 14,8$ & $13,5 \pm 17$ \\
\hline Estancia en UCI tras el trasplante (días) & $31,5 \pm 25,4$ & \\
\hline Estancia hospitalaria tras el trasplante (días) & & \\
\hline
\end{tabular}

ACM: asistencia circulatoria mecánica; IC95\%: intervalo de confianza del 95\%; UCI: unidad de cuidados intensivos.

La tasa de incidencia es el número de pacientes con un evento cada 1.000 días-paciente de ingreso posoperatorio después del trasplante.

Los valores expresan $\mathrm{n}(\%)$ o media \pm desviación estándar. 


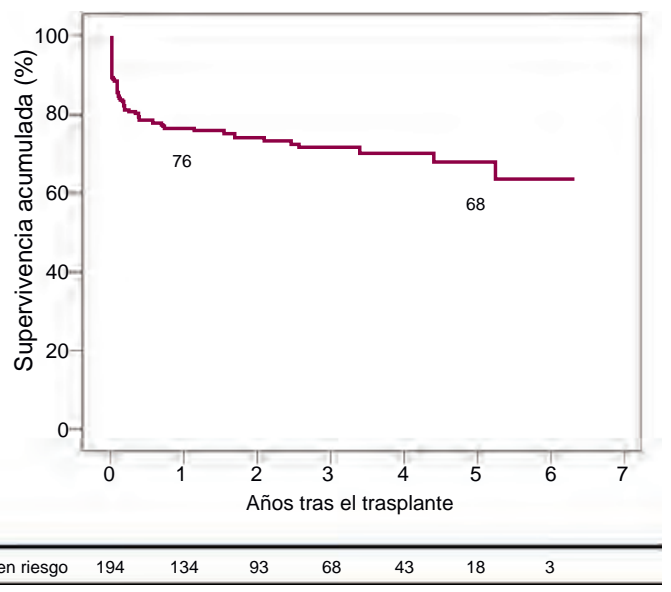

Figura 4. Supervivencia posoperatoria a largo plazo de los 194 pacientes sometidos a trasplante cardiaco asistidos con balón de contrapulsación intraaórtico.

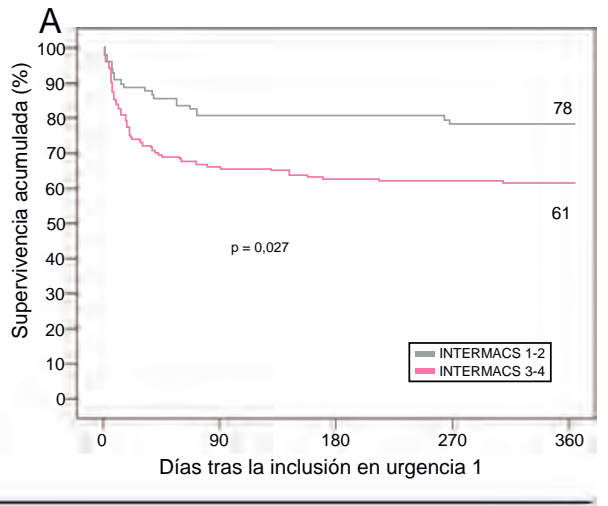

\begin{tabular}{lccccc}
\hline Pacientes en riesgo & & & & & \\
INTERMACS 1-2 & 177 & 116 & 108 & 107 & 104 \\
INTERMACS 3-4 & 98 & 79 & 74 & 66 & 63 \\
\hline
\end{tabular}

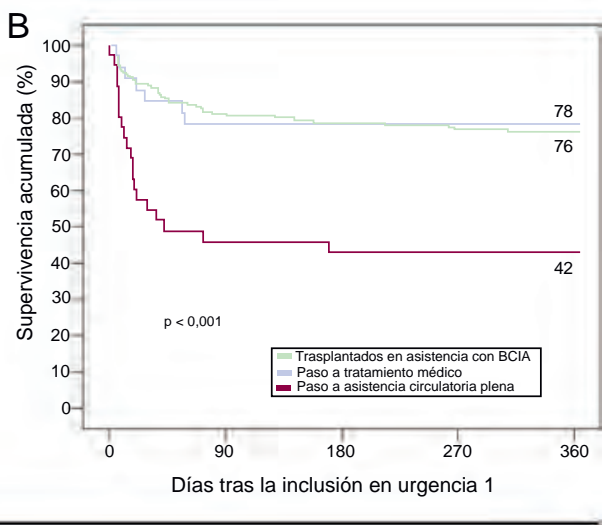

\begin{tabular}{lccccc}
\hline Pacientes en riesgo & & & & & \\
Trasplantados & 194 & 155 & 144 & 136 & 130 \\
Paso a tratamiento médico & 31 & 25 & 25 & 25 & 24 \\
Paso a asistencia circulatoria plena & 34 & 16 & 15 & 13 & 13 \\
\hline
\end{tabular}

Figura 5. A: supervivencia el primer año tras la inclusión en la lista de espera en urgencia 1 según el perfil de INTERMACS. B: supervivencia el primer año tras la inclusión en la lista de espera en urgencia 1 según el objetivo de la asistencia con balón de contrapulsación intraaórtico. BCIA: balón de contrapulsación intraaórtico; INTERMACS: Interagency Registry for Mechanically Assisted Circulatory Support. trasplante específicas de estos 3 subgrupos de pacientes se presentan en la figura del material suplementario.

\section{DISCUSIÓN}

En este registro nacional, se estudian los resultados clínicos previos y posteriores al trasplante en 281 pacientes adultos incluidos en lista de espera para TxC asistidos con BCIA en 16 centros de España entre 2010 y 2015 . Aproximadamente, más de 2 tercios de ellos recibieron un corazón de donante durante la asistencia con BCIA, con una media de espera de $\sim 10$ días. Las tasas de supervivencia a 30 días y 1 y 5 años tras el trasplante fueron de un 88 , un 76 y un $68 \%$ respectivamente. Se obtuvieron peores resultados en los candidatos que debieron pasar del BCIA a un dispositivo de ACM completa a causa de la progresión del deterioro hemodinámico mientras estaban a la espera del trasplante. En este subgrupo, la supervivencia a 1 año tras la inclusión en la lista de espera urgente se redujo a $\sim 43 \%$.

El BCIA es el método más ampliamente disponible en todo el mundo para proporcionar ACM. La principal ventaja de este dispositivo es su fácil implante percutáneo, que permite iniciar inmediatamente la asistencia circulatoria a la cabecera del paciente. El tratamiento con BCIA reduce discretamente la resistencia vascular sistémica y produce un discreto aumento del gasto cardiaco hasta $\sim 1 \mathrm{l} / \mathrm{min}$, siempre que el ventrículo izquierdo en insuficiencia conserve cierta contractilidad que permita aplicar la contrapulsación ${ }^{7}$. Estos efectos hemodinámicos aumentan la perfusión periférica, con lo que se atenúa el daño experimentado por los órganos diana ${ }^{8}$. En los pacientes con cardiopatía isquémica, la potenciación diastólica de la presión aórtica que se produce al hinchar el BCIA aumenta de manera significativa el flujo sanguíneo coronario ${ }^{9}$. En los pacientes con alto riesgo sometidos a cirugía de revascularización coronaria, el inicio preoperatorio de la asistencia circulatoria con BCIA reduce significativamente el riesgo de insuficiencia renal posoperatoria ${ }^{10}$.

La mejora hemodinámica aportada por el tratamiento con BCIA suele bastar para la estabilización inicial de muchos pacientes con IC avanzada y signos de bajo gasto cardiaco, en especial en el contexto de una cardiopatía isquémica, como puente para la toma de una decisión o hasta la recuperación. Sin embargo, el BCIA rara vez se emplea como puente directo al TxC, principalmente por 2 razones. En primer lugar, la asistencia circulatoria parcial que proporciona el dispositivo con frecuencia resulta insuficiente para los pacientes con gran deterioro hemodinámico o insuficiencia ventricular derecha. De hecho, algunos datos recientes indican que el tratamiento con BCIA no modifica la supervivencia de los pacientes en shock cardiogénico como complicación de un infarto agudo de miocardio ${ }^{11}$. En segundo lugar, conceptualmente, el BCIA tiene como finalidad el tratamiento a corto plazo, por lo que su utilidad como puente al TxC se ve limitada por la probabilidad de que el paciente pueda disponer de un donante apropiado en los primeros días tras el inicio de la asistencia circulatoria. Se ha propuesto que el implante del BCIA a través de un acceso humeral es una alternativa segura que permite una asistencia circulatoria más prolongada que la obtenida con el implante clásico por vía femoral $^{12}$; sin embargo, este abordaje rara vez se usa en la práctica clínica actual.

Es de destacar que, incluso en un contexto en el que los tiempos de espera para el TxC sean cortos, como ocurre en la eficiente red de distribución de órganos de donantes de España ${ }^{1}$, casi un tercio de los pacientes estudiados no dispusieron de un donante durante el periodo de uso del BCIA. Conviene señalar que la supervivencia de los candidatos incluidos en lista de espera con un perfil INTERMACS 1 o 2 mostró un empeoramiento significativo ${ }^{13}$, lo cual probablemente indique que la asistencia brindada por el BCIA era 
insuficiente en muchos de ellos. El pronóstico fue especialmente negativo para los candidatos que pasaron del BCIA a la ACM plena (en la mayoría de los casos con dispositivos de uso transitorio) a causa de la progresión del deterioro clínico mientras se esperaba el TxC, a pesar de que una parte significativa de ellos pasaron al nivel más alto de prioridad en la lista de espera (urgencia 0) y después recibieron un trasplante.

Los resultados tras el trasplante en los pacientes en los que se pudo usar con éxito el BCIA como puente al TxC fueron aceptables, puesto que resultaron comparables a los descritos en la cohorte histórica completa de los receptores de TxC de España ${ }^{14}$. La incidencia de complicaciones posoperatorias tempranas y las causas de muerte fueron, en general, similares a las esperadas, con la notable excepción de una tasa anormalmente alta de fallos primarios del injerto, que podría ser atribuible al uso frecuente de donantes añosos con tiempos de isquemia prolongados; sin embargo, debe señalarse que fueron los investigadores locales quienes tuvieron la responsabilidad de registrar los eventos adversos clínicos, por lo que no puede descartarse por completo un sesgo de observación positivo.

Aunque en un modelo multivariable derivado del registro United Organ Network for Organ Sharing ${ }^{4}$ se ha identificado que la asistencia circulatoria preoperatoria con un BCIA es un factor de riesgo de muerte tras el trasplante, en un estudio unicéntrico europeo, centrado en este aspecto específico, no se confirmó una influencia negativa independiente del BCIA en los resultados posteriores al trasplante ${ }^{5}$. Desde un punto de vista clínico, parece improbable que el dispositivo en sí influya de manera significativa en el riesgo quirúrgico del trasplante; más bien da la impresión de que es más probable que las discrepancias entre los datos presentados reflejen un perfil de riesgo heterogéneo de los participantes estudiados. De hecho, la literatura médica previa indica que el estado clínico del receptor, más que el tipo de asistencia circulatoria utilizado, probablemente sea el más firme factor predictivo de los resultados posoperatorios tras el $\mathrm{TxC}^{13}$.

Otra característica notable del estudio es que casi la mitad de los pacientes incluidos en lista de espera en urgencia 1 estando en asistencia con BCIA no eran casos nuevos, sino que ya habían estado incluidos en lista no urgente. Dada la creciente escasez de donantes y los tiempos de espera para el TxC, el implante semielectivo de un DAV duradero como puente al TxC para los pacientes con dependencia de inotrópicos que empiezan a deteriorarse o incluso que se mantienen estables, se está introduciendo como estrategia más razonable para romper el círculo vicioso que actualmente favorece un aumento progresivo de la proporción de donantes de corazón que se destinan a candidatos urgentes cada año en España ${ }^{14}$. Los datos recientes del registro de la International Society for Heart and Lung Transplantation indican un efecto protector de la asistencia preoperatoria con DAV de flujo continuo en los resultados tras el trasplante, que probablemente se deba a los efectos favorables del dispositivo en la función de los órganos diana, la fragilidad y el estado nutricional ${ }^{15}$. Respalda este argumento un reciente estudio unicéntrico estadounidense ${ }^{16}$ que ha mostrado un mejor resultado clínico en los candidatos que pasaron de usar dispositivos de corta duración a dispositivos de larga duración y luego recibieron un trasplante que en los candidatos cuyo TxC se practicó directamente mientras estaban en ACM transitoria.

A la vista de esta situación, las autoridades sanitarias españolas han remodelado recientemente los criterios de prioridad en las listas de espera de los candidatos a un TxC. Desde 2017, la asistencia con BCIA ha dejado de considerarse una indicación urgente, y los criterios exigidos para la inclusión en la lista de espera para trasplante urgente de los pacientes tratados con oxigenador extracorpóreo de membrana o DAV transitorios son más restrictivos ${ }^{17}$.
En la presente cohorte, la tasa diaria general de eventos adversos clínicos asociados con el uso de BCIA se aproximó a un $4 \%$. Esta tasa de incidencia es inferior a la tasa descrita de eventos adversos de los candidatos tratados con ACM transitoria ${ }^{6}$, pero aun así parece ser más alta que la observada en los portadores de DAV duraderos $^{18}$. La mayor parte de los eventos adversos clínicos registrados en el estudio, como infección, hemorragia o complicaciones tromboembólicas, pueden considerarse en cierta medida inherentes al crítico estado clínico de los pacientes estudiados, pero en algunos casos podrían verse favorecidos también por el tratamiento con BCIA.

La tasa acumulada de cambio del BCIA a causa de complicaciones directamente atribuibles al dispositivo, como isquemia de las extremidades, disfunción mecánica o dolor resistente al tratamiento, fue del 7,8\% durante todo el periodo de asistencia circulatoria; otros estudios han observado una tasa de complicaciones asociadas con el uso del BCIA de entre el 2,6 y el 13\% ${ }^{19,20}$. Estos datos ponen de manifiesto que el tratamiento con BCIA, a pesar de su aparente sencillez y amplia disponibilidad, no es en absoluto inocuo.

\section{Limitaciones}

Este estudio tiene unas pocas limitaciones. En primer lugar, al tratarse de un estudio observacional, está expuesto a posibles sesgos de información, selección, observación y confusión. Segundo, la naturaleza de esta investigación es básicamente descriptiva, más que analítica; la falta de un grupo de control paralelo formado por candidatos a TxC emparejados por sus características pero que recibieron un tratamiento médico impide extraer conclusiones sólidas respecto a un posible efecto independiente del BCIA en la supervivencia previa y posterior al trasplante. Tercero, la población del estudio es heterogénea, puesto que se incluyó a pacientes de 16 centros diferentes, con unos protocolos y una experiencia clínica diversas y específicas de cada centro. Cuarto, todos los pacientes fueron tratados en el contexto de la Organización Nacional de Trasplantes de España, que se ha caracterizado históricamente por unos tiempos de espera extraordinariamente cortos para las indicaciones de TxC urgente; por consiguiente, la validez externa de estas observaciones es dudosa, y los resultados podrían no ser directamente extrapolables a otros países. Por último, debe reconocerse que el estudio se centró en un periodo reciente (aunque, hasta cierto punto, también pasado) de la evaluación en este campo; teniendo en cuenta los recientes cambios introducidos en los criterios clínicos exigidos para la inclusión en lista de espera para TxC urgente en España, cabe presumir que el uso del BCIA como puente directo al TxC pasará a ser menos frecuente en los próximos años.

\section{CONCLUSIONES}

El BCIA continúa siendo un método ampliamente disponible para brindar asistencia circulatoria inicial a los candidatos a TxC en estado crítico. En los casos en que se alcanza la estabilización clínica, el BCIA puede usarse como puente directo para el TxC urgente siempre que los tiempos de espera previstos sean cortos, con unos resultados aceptables tras el trasplante. Sin embargo, en los casos en que se produce un deterioro hemodinámico profundo a pesar del tratamiento con BCIA, debe considerarse la posible conveniencia de implantar un dispositivo de ACM completa.

A pesar de su aparente simplicidad, el tratamiento con BCIA no es una técnica inocua, puesto que se asocia con un riesgo significativo de eventos adversos clínicos, como isquemia de extremidades, tromboembolia, disfunción del dispositivo e infección. 
En los próximos años, el aumento del tiempo de permanencia en lista de espera y las restricciones en las indicaciones para el TxC urgente probablemente limiten la utilidad del BCIA como puente directo al TxC en España.

\section{AGRADECIMIENTOS}

Los autores agradecen a la Organización Nacional de Trasplantes la financiación para esta investigación.

\section{FINANCIACIÓN}

E. Barge-Caballero recibió una subvención de investigación de la Fundación Mutua Madrileña, España (XI anuncio anual año 2014), que proporcionó la financiación para este artículo.

Algunos de los autores de este manuscrito forman parte del CIBERCV (Centro de Investigación Biomédica en Red de Enfermedades Cardiovasculares), Instituto de Salud Carlos III, Ministerio de Economía y Competitividad de España.

\section{CONFLICTO DE INTERESES}

E. Barge-Caballero recibió una subvención académica de St. Jude Medical, no relacionada con esta investigación.

\section{¿QUÉ SE SABE DEL TEMA?}

- El empleo del BCIA como puente directo al TxC está condicionado por el hecho de que debe disponerse de un donante adecuado para el paciente en un plazo de pocos días tras el inicio de la asistencia.

- A diferencia de lo que ocurre en otros países, en España el corto tiempo de espera para los candidatos en lista de espera urgente ha favorecido históricamente el empleo del BCIA como puente directo al TxC.

\section{¿QUÉ APORTA DE NUEVO?}

- Este estudio proporciona una descripción sistemática de unas características únicas respecto a los resultados previos y posteriores al trasplante en los pacientes asistidos con BCIA con el objetivo primario como puente al trasplante en España.

- El estudio pone de manifiesto que el BCIA ha sido una opción viable como puente al TxC urgente para los pacientes de España, con unos resultados posoperatorios razonables. Sin embargo, el aumento que se está produciendo en el tiempo en lista de espera y que las indicaciones para el TxC urgente se hayan restringido probablemente limiten la utilidad de esta estrategia en los próximos años.

\section{ANEXO. MATERIAL SUPLEMENTARIO}

Se puede consultar material adicional a este artículo en su versión electrónica disponible en https://doi.org/10.1016/j.recesp. 2018.07.006

\section{BIBLIOGRAFÍA}

1. Unverzagt S, Buerke M, De Waha A, et al. Intra-aortic balloon pump counterpulsation (IABP) for myocardial infarction complicated by cardiogenic shock. Cochrane Database Syst Rev. 2015;3:CD007398.

2. Matesanz R. Spain: a leader in harvesting hearts for transplantation. Circulation. 2007; $115: f 45-f 46$.

3. Barge-Caballero E, Almenar-Bonet L, Villa-Arranz A, et al. Impact of short term mechanical circulatory support with extracorporeal devices on postoperative outcomes after emergency heart transplantation: data from a multi-institutional Spanish cohort. Int J Cardiol. 2014;176:86-93.

4. Weiss ES, Allen JG, Arnaoutakis GJ, et al. Creation of a quantitative recipient risk index for mortality prediction after cardiac transplantation (IMPACT). Ann Thorac Surg. 2011;92:914-921.

5. Gjesdal O, Gude E, Arora S, et al. Intra-aortic balloon counterpulsation as a bridge to heart transplantation does not impair long-term survival. Eur J Heart Fail. 2009;11:709-714.

6. Barge-Caballero E, Almenar-Bonet L, Gonzalez-Vilchez F, et al. Clinical outcomes of temporary mechanical circulatory support as a direct bridge to heart transplantation. A nationwide Spanish registry. Eur J Heart Fail. 2017. http://dx.doi.org/ 10.1002/ejhf.956. Consultado 2 Jul 2018.

7. Prondzinsky R, Unverzagt S, Russ M, et al. Hemodynamic effects of intra-aortic balloon counterpulsation in patients with acute myocardial infarction complicated by cardiogenic shock: the prospective, randomized IABP shock trial. Shock. 2012;37:378-384.

8. Parissis H, Graham V, Lampridis S, et al. IABP: history-evolution-pathophysiologyindications: what we need to know. J Cardiothorac Surg. 2016;11:122.

9. Takeuchi M, Nohtomi Y, Yoshitani H, et al. Enhanced coronary flow velocity during intra-aortic balloon pumping assessed by transthoracic Doppler echocardiography. J Am Coll Cardiol. 2004;43:368-376.

10. Wang J, Yu W, Gao J, et al. Preoperative prophylactic Intraaortic Balloon Pump Reduces the Incidence of Postoperative Acute Kidney Injury and Short-Term Death of High-Risk Patients Undergoing Coronary Artery Bypass Grafting: A Meta-Analysis of 17 Studies. Ann Thorac Surg. 2016;101:2007-2019.

11. Ahmad Y, Sen S, Shun-Shin MJ, et al. Intraaortic balloon pump therapy for myocardial infarction: a meta-analysis. JAMA Intern Med. 2015;175:931-939.

12. Estep JD, Cordero-Reyes AM, Bhimaraj A, et al. Percutaneous placement of an intraaortic balloon pump in the left axillary/subclavian position provides safe, ambulatory long-term support as bridge to heart transplantation. JACC Heart Fail. 2013;1:382-388

13. Barge-Caballero E, Segovia-Cubero J, Almenar-Bonet L, et al. Preoperative INTERMACS profiles determine postoperative outcomes in critically ill patients undergoing emergency heart transplantation: analysis of the Spanish National Heart Transplant Registry. Circ Heart Fail. 2013;6:763-772.

14. González-Vílchez F, Gómez-Bueno M, Almenar L, et al.Spanish Heart Transplant Registry. 28th Official Report of the Spanish Society of Cardiology Working Group on Heart Failure (1984-2016). Rev Esp Cardiol. 2017;70:1098-1109.

15. Lund LH, Khush KK, Cherikh WS, et al. The Registry of the International Society for Heart and Lung Transplantation: Thirty-fourth Adult Heart Transplantation Report-2017; Focus Theme: Allograft ischemic time. J Heart Lung Transplant. 2017;36:1037-1046

16. Yoshioka D, Li B, Takayama $\mathrm{H}$, et al. Outcome of heart transplantation after bridgeto-transplant strategy using various mechanical circulatory support devices. Interact Cardiovasc Thorac Surg. 2017;25:918-924.

17. Barge-Caballero E, Gonzalez-Vilchez F, Farrero-Torres M, Segovia-Cubero J. Selection of the Best of 2017 in Cardiac Transplant and Ventricular Assist Devices. Rev Esp Cardiol. 2018;71:300-301.

18. Kirklin J, Cantor R, Mohacsi P, et al. First Annual IMACS Report: A global International Society for Heart and Lung Transplantation Registry for Mechanical Circulatory Support. J Heart Lung Transplant. 2016;35:407-412.

19. Ferguson JJ, Cohen M, Freedman RJ, et al. The current practice of intra-aortic balloon counterpulsation: results from the Benchmark Registry. J Am Coll Cardiol. 2001;38:1456-1462.

20. Valente S, Lazzeri C, Crudeli E, et al. Intraaortic balloon pump: incidence and predictors of complications in the Florence Registry. Clin Cardiol. 2012;35:200-204. 